\title{
The Influence of Nozzle Geometry on Corner Flows in Supersonic Wind Tunnels
}

\author{
Kshitij Sabnis* and Holger Babinsky ${ }^{\dagger}$ \\ Department of Engineering, University of Cambridge, Cambridge, CB2 1PZ, UK \\ Daniel S. Galbraith ${ }^{\ddagger}$ and John A. Benek ${ }^{\S}$ \\ Air Force Research Laboratory, Wright-Patterson AFB, Ohio, United States
}

\begin{abstract}
In supersonic flows, the separation in streamwise corners is a significant and widely encountered problem which can not be reliably predicted with the numerical methods commonly used in industry. The few previous studies on this topic have suggested conflicting corner flow topologies. Experiments of supersonic flow are typically conducted in wind tunnels with rectangular cross-sections, which use either a symmetric (full) or asymmetric (halfliner) nozzle configuration. However, the effect of the nozzle arrangement on the corner flow itself is not known. This paper examines the influence of nozzle geometry on the corner regions of a Mach 2.5 flow using a joint experimental-computational approach. The full setup and half-liner configuration are shown to produce different corner flow structures. The corner regions of the full setup and top corners of the half-liner exhibit thin sidewall boundary layers and a single primary vortex on the floor or ceiling. Meanwhile, the bottom corners of the half-liner configuration contain thick sidewall boundary layers and a counter-rotating vortex pair. Considerable vertical velocities are measured within the sidewall boundary layers. These are directed towards the tunnel centre-height for the full setup and downwards with the half-liner. The differences in sidewall cross flows between the two nozzle arrangements are likely due to distinct pressure distributions in the nozzle, where the secondary flows are set up. Measurements suggest that these nozzle-dependent transverse flows are responsible for the differences in corner flowfield between the two configurations. The proposed mechanism also explains observed differences in corner flow topology between previous studies in the literature; nozzle geometry therefore appears to be the dominant influence on corner flows in supersonic wind tunnels.
\end{abstract}

\section{Nomenclature}

shape factor

Mach number

pressure

temperature

streamwise velocity

floor-normal velocity

spanwise velocity

streamwise coordinate, measured from nozzle exit

floor-normal coordinate, measured from tunnel floor

spanwise coordinate, measured from tunnel centre span

*PhD Student, Department of Engineering, University of Cambridge.

${ }^{\dagger}$ Professor in Aerodynamics, Department of Engineering, University of Cambridge, AIAA Associate Fellow.

${ }^{\ddagger}$ Engineer, Computational Sciences Centre, AFRL Aerospace Systems Directorate.

$\S$ Director, Computational Sciences Centre, AFRL Aerospace Systems Directorate, AIAA Fellow. 
$\delta \quad$ boundary layer thickness (99\% of freestream velocity)

$\delta^{*} \quad$ displacement thickness

$\theta \quad$ momentum thickness

\section{Subscript}

$i \quad$ equivalent incompressible quantity

$0 \quad$ stagnation property

0s settling chamber stagnation property

$\infty \quad$ freestream property

Superscript

$+\quad$ quantity expressed in non-dimensional wall units

\section{Abbreviations}

CAD computer-aided design

CFD computational fluid dynamics

HLLC Harten-Lax-van Leer-Contact

LDV laser Doppler velocimetry

LES large eddy simulation

RANS Reynolds-averaged Navier Stokes

SSOR symmetric successive over-relaxation

\section{Introduction}

Streamwise corners are a common feature in many high-speed flows, both internal - such as in the rectangular inlets of supersonic aircraft or the working sections of high speed wind tunnels - and external, in the case of aircraft wing-body junctions. The flow in these geometries is both unavoidable and often problematic. The corner corresponds to the intersection of two viscous surfaces, and hence the boundary layer experiences a momentum deficit. This region of low momentum flow is highly susceptible to adverse pressure gradients, and so tends to exhibit separation, with its associated problems, furthest upstream. This can have a substantial impact on the overall flowfield; for example, corner effects in the wing-body junction are estimated to contribute $4-6 \%$ to the total drag of aircraft and the range of supersonic fighter aircraft is thought to be reduced by as much as $9 \%$ owing to the flows in inlet corners. ${ }^{1}$

Despite the significance and pervasiveness of the corner flow issue, there are still no consistently successful techniques to mitigate corner separation, apart from (possibly) targeted surface bleed. ${ }^{2,3}$ The difficulties in alleviating these issues can arguably be directly attributed to our lack of understanding of the complicated flow physics in streamwise corners, and of their interactions with shock waves.

The substantial impact of these effects on the flowfield, even away from the corners, is problematic in its own right. There is growing evidence ${ }^{4-7}$ that these features result in significant departures from twodimensionality elsewhere in the flow, and so cannot simply be neglected. More recently, the relationship between separation in corners and the overall flowfield has been studied; this mechanism is physically based on the compression and expansion waves emanating from the corner due to the displacement effect caused by separation in this region. ${ }^{8}$ However, it is still not possible to reliably predict the corner separation with standard techniques used in industry.

A number of researchers have investigated the streamwise corners of supersonic flows. ${ }^{9-14}$ Whilst all authors have identified vortices in the corner region, their results do not appear to be entirely consistent. Figure 1a shows measurements from the corner of a duct within a Mach 3.9 tunnel core flow; ${ }^{9-11}$ the vortex structure is symmetric about the corner bisector. However, in a different study, a Mach 2 corner flow generated using a symmetric nozzle configuration ${ }^{13,14}$ (Figure 1b) shows a single primary vortex. Yet another flow topology is found in figure 1c, which was obtained in a Mach 2.75 flow with an asymmetric half-liner setup; ${ }^{12}$ here, there is a vortex pair sitting on the tunnel floor.

The three separate vortex structures in figure 1 implies that there is no single 'typical' corner flow. Moreover, it is not clear which factors influence the flow topology in this region. It has been suggested that the flow features can be related to characteristics of the nozzle geometry ${ }^{12,13}$ or the aspect ratio of the 
tunnel, ${ }^{12}$ but no coherent mechanism has been proposed to explain them. Furthermore, the distinct corner flow structures have not been directly compared with one another till now and the reasons for the differences between them are not well understood. This is compounded by an inability to easily isolate possible causes for the observed variations from one another since previous investigations were performed under a variety of different conditions (facilities, Mach numbers, nozzle configurations, tunnel aspect ratios, ...).

The supersonic wind tunnel at Cambridge University Engineering Department has the option of operating in both a symmetric nozzle (full setup) and a half-liner configuration, analogous to the arrangements in figures $1 \mathrm{~b}$ and $1 \mathrm{c}$ respectively. This provides a rare opportunity to test the influence of nozzle geometry on corner flows in a single facility.

The combined experimental-computational study presented in this paper focuses measurements on the streamwise corners of a Mach 2.5 flow without an adverse pressure gradient. Two different tunnel configurations (full setup and half-liner) are used, which enables insight into the effect of nozzle geometry. Careful collation of flow characterisation data provides a quantiative assessment of flow quality and allows numerical simulations to accurately represent the physical tunnel. A mechanism is suggested for the generation of observed secondary flows in the sidewall boundary layers, which appear to influence the structure of the corner flows themselves.

a)
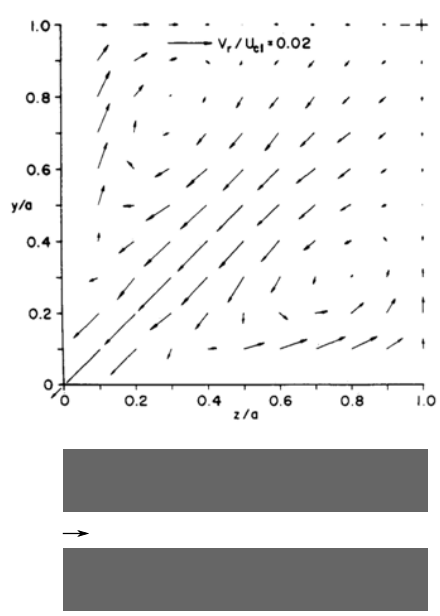

b)
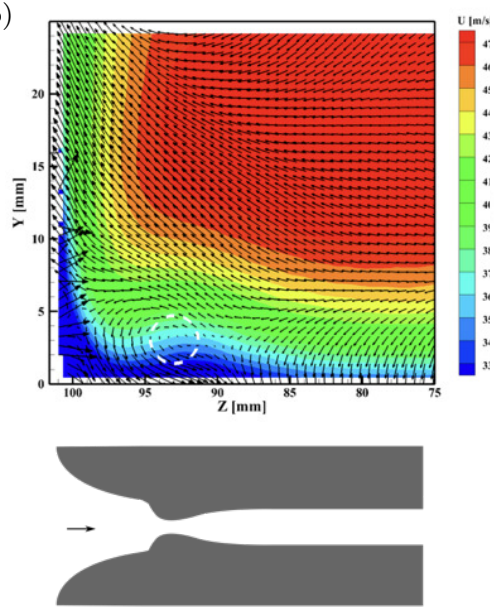
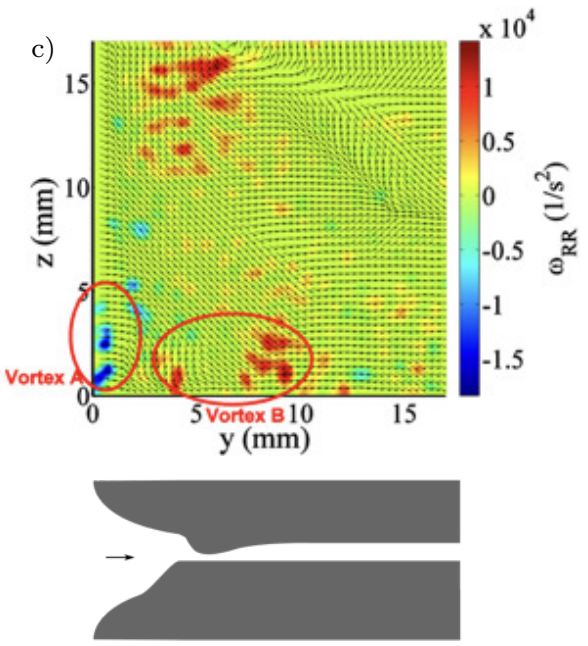

Figure 1. Measured corner flows from the literature: a) Davis and Gessner (1989) ${ }^{10}$ in a square duct; b) Peltier et al. $(2018)^{14}$ with a symmetric nozzle; c) Morajkar et al. $(2015)^{12}$ using an asymmetric nozzle setup.

a)

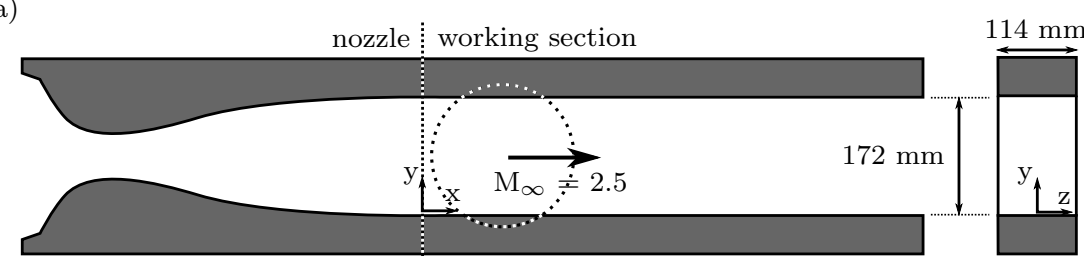

b)

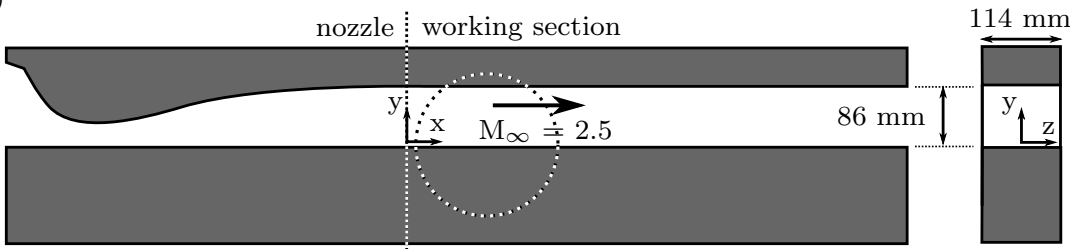

Figure 2. Tunnel setup for a) the full and b) the half-liner configurations. The dashed circle corresponds to a window in the tunnel wall providing optical access. 


\section{Research Methodology}

Experiments are performed in Supersonic Wind Tunnel No. 1 at Cambridge University Engineering Department. This is a blow-down wind tunnel, driven by a high-pressure reservoir. The facility is capable of operating at Mach numbers between 0.7 and 3.5, depending on the installed nozzle configuration; for this study, the nominal freestream Mach number is fixed at $\mathrm{M}_{\infty}=2.5$. The tunnel has the option of operating in both a full set-up and a half-liner configuration, as depicted in figure 2. This offers the particular benefit for this study that two corner boundary layers with quite different 'histories' can be compared.

The stagnation pressure is set to avoid tunnel unstart at $308 \pm 1 \mathrm{kPa}$ and the operating stagnation temperature is measured as $285 \pm 5 \mathrm{~K}$; this corresponds to a unit Reynolds number of approximately $31 \times 10^{6}$ $\mathrm{m}^{-1}$. An empty shock-free wind tunnel configuration is used in this study, and the measurements focus on the turbulent, naturally-grown boundary-layers on the tunnel's floor and sidewalls. These are approximately $7-8 \mathrm{~mm}$ thick, and have a Reynolds number based on incompressible displacement thickness of around $\operatorname{Re}_{\delta_{i}^{*}}=30,000$.

The rectangular working section of the tunnel has a width of $114 \mathrm{~mm}$, and a height of $172 \mathrm{~mm}$ and $86 \mathrm{~mm}$ for the full and half-liner configurations respectively. The coordinate system convention is shown in figure 2 . $x$ represents the streamwise direction, as measured from the end of the nozzle; $y$ indicates the floor-normal direction, with $y=0 \mathrm{~mm}$ set at the tunnel floor; $z$ is the spanwise coordinate measured from the centre span, such that $z= \pm 57 \mathrm{~mm}$ correspond to the tunnel sidewalls.

The precise tunnel geometry is used to calibrate computational models alongside the design CAD model. The 'as-installed' shape is measured by by securing a dial test indicator to a three component traverse, and scanning along the target surface. This allows the profile of the tunnel floor to be mapped to within 0.01 $\mathrm{mm}$. Figure 3 shows an example for the half-liner configuration; there are variations on the order of 0.1 $\mathrm{mm}$, or $1.5 \%$ of a boundary layer thickness, from a designed linear divergence (to provide boundary layer relief). The tilt in the spanwise direction is $(0.06 \pm 0.10)^{\circ}$, this value is within the alignment accuracy of the measurement apparatus; the surface can therefore be considered flat in this direction, to within experimental error.

The inflow pressure uniformity is quantified by measuring the stagnation pressure distribution at the exit of the settling chamber, upstream of the nozzle. A Pitot rake is installed here, and the probes are connected to a differential pressure transducer NetScanner 9116. The rated transducer error is $0.05 \%$; this was verified by comparison with a mercury barometer. There is a further uncertainty contribution from determining the atmospheric pressure (to convert gauge to absolute pressures) and so the overall measurement error is $0.1 \%$.

Within the working section, a z-type Schlieren system with a horizontal knife-edge enables visualisation of density gradients and allows flow features to be identified. In addition, steady-state surface pressure measurements are performed using $0.3 \mathrm{~mm}$ diameter static pressure taps (error: $\pm 1 \%$ ). ${ }^{15}$ The taps are located across the tunnel floor and sidewall, allowing the pressure distributions over these surfaces to be measured.

The streamwise and floor-normal flow velocities, $u$ and $v$ respectively, are measured by two-component LDV. The flow is seeded with paraffin in the settling chamber; previous measurements of particle lag through a normal shock have placed the seeding droplet diameter in the range $200-500 \mathrm{~nm} .{ }^{15}$ The measured velocities have an error of $1 \%$ and $14 \%$ for $u$ and $v$ respectively; there are contributions from the rate of seeding
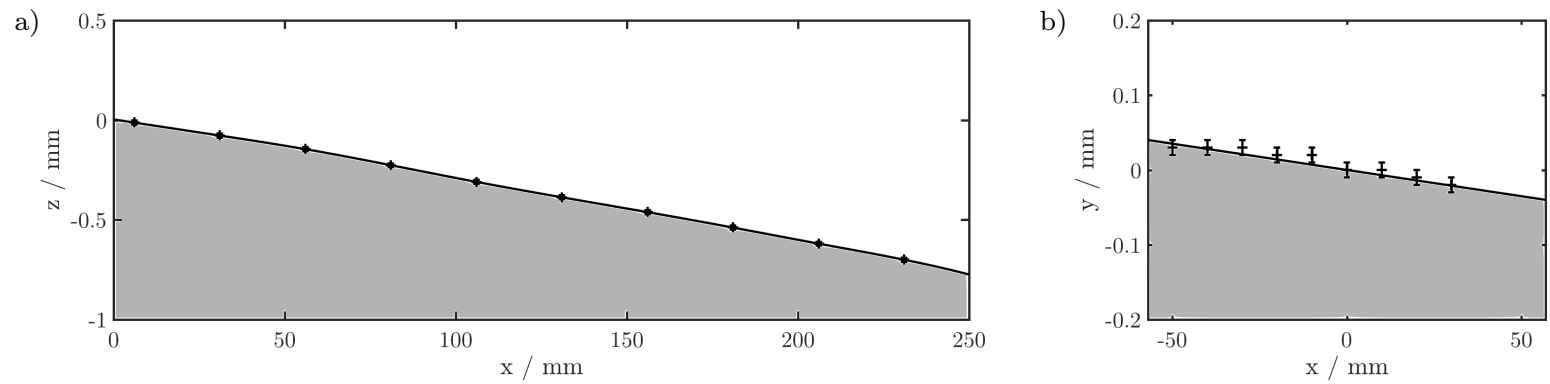

Figure 3. Measurement of tunnel geometry a) in the streamwise direction at $z=0 \mathrm{~mm}$, and b) in the spanwise direction at $x=0 \mathrm{~mm}$. 
a)

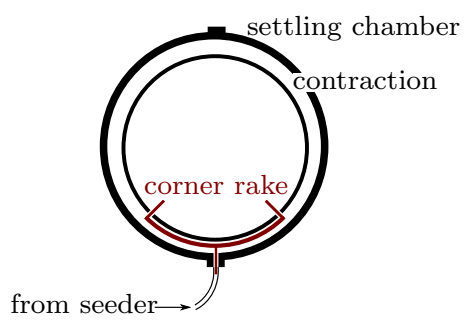

b)

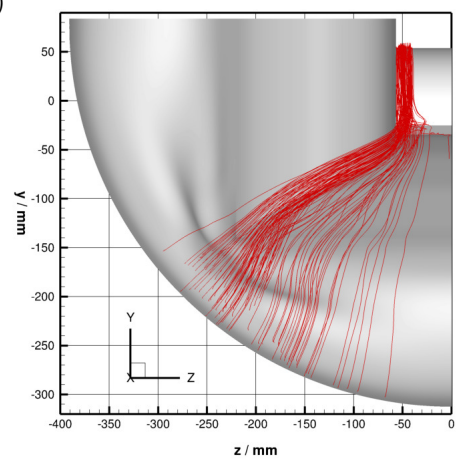

c)

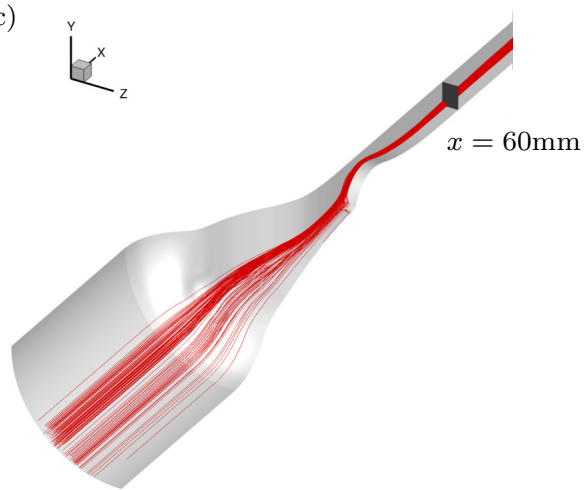

Figure 4. a) Schematic cross-sectional view of corner seeding system, located in the tunnel's settling chamber. Seeding particles are introduced to the flow from the corner rakes. b) Simulated streamlines traced upstream into the settling chamber. c) Isometric view of simulated corner flow streamlines illustrating the 'seeding plane' in the corner region in the working section.

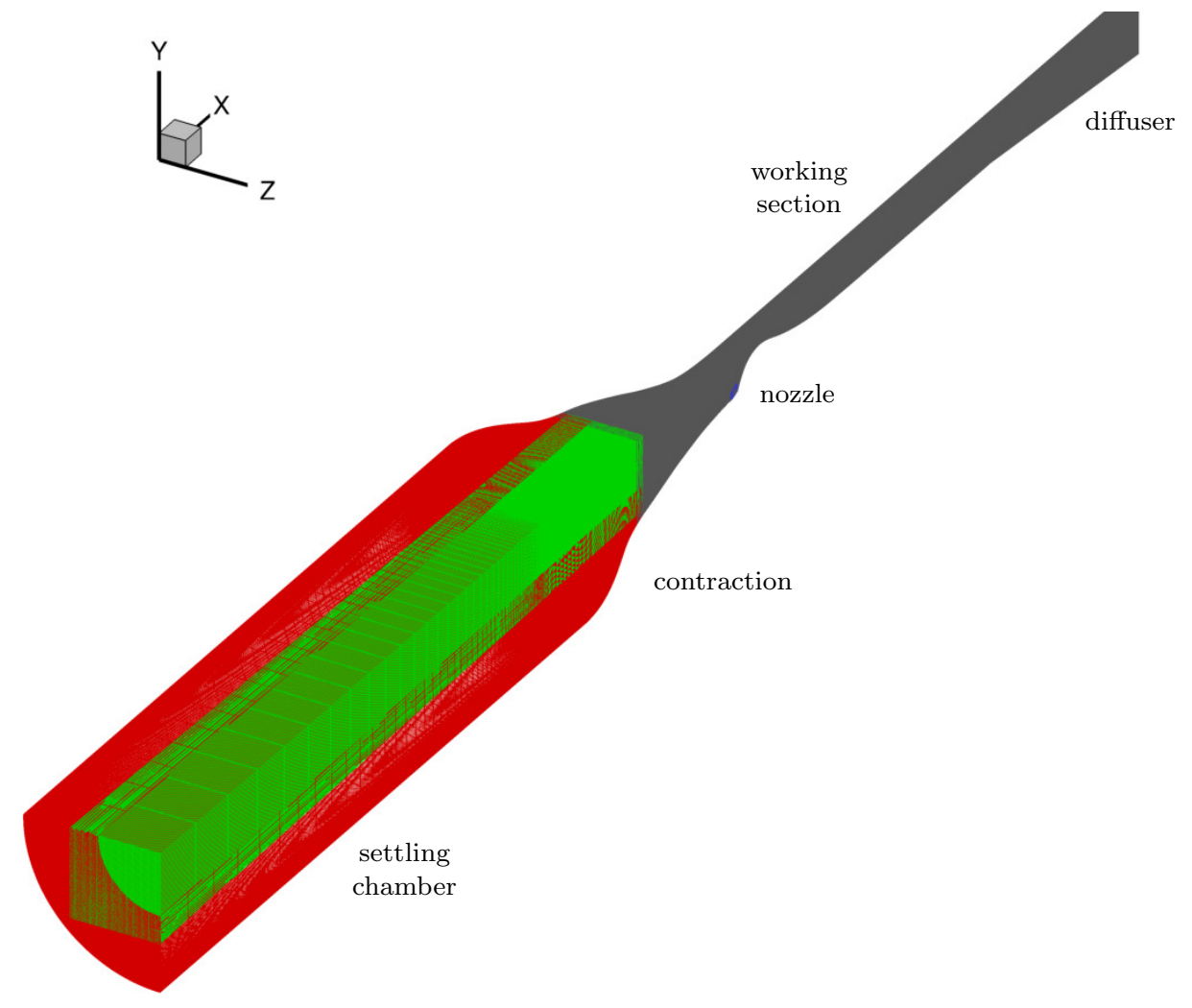

Figure 5. Fine mesh used in computations to simulate physical tunnel. 
a)

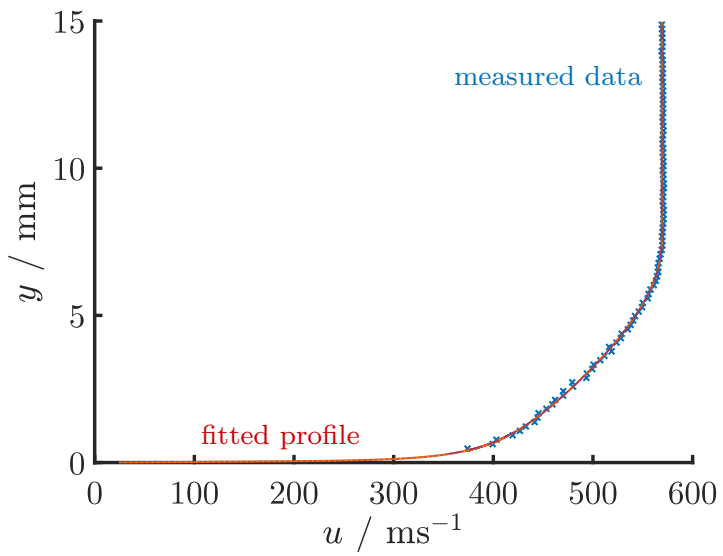

b)

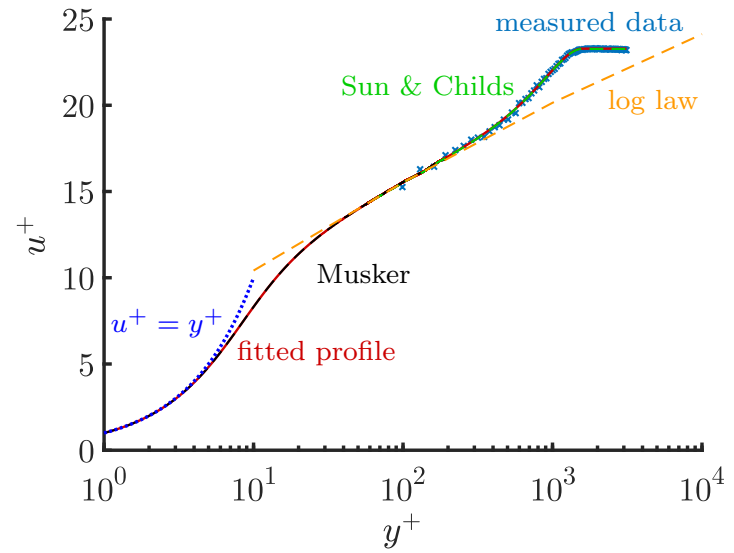

Figure 6. Boundary layer LDV data and fitted profile in a) linear, and b) $\log -\log$ form. Measurements performed at $x=60 \mathrm{~mm}, z=0 \mathrm{~mm}$. In log-log form, the data are presented in non-dimensional wall units, $u^{+}=\frac{u}{u_{\tau}}$ and $y^{+}=\frac{y u_{\tau}}{\nu_{w}}$. The error bars are contained within the symbol size with $\Delta u=5 \mathrm{~ms}^{-1}$ and $\Delta y=0.1$ $\mathrm{mm}$

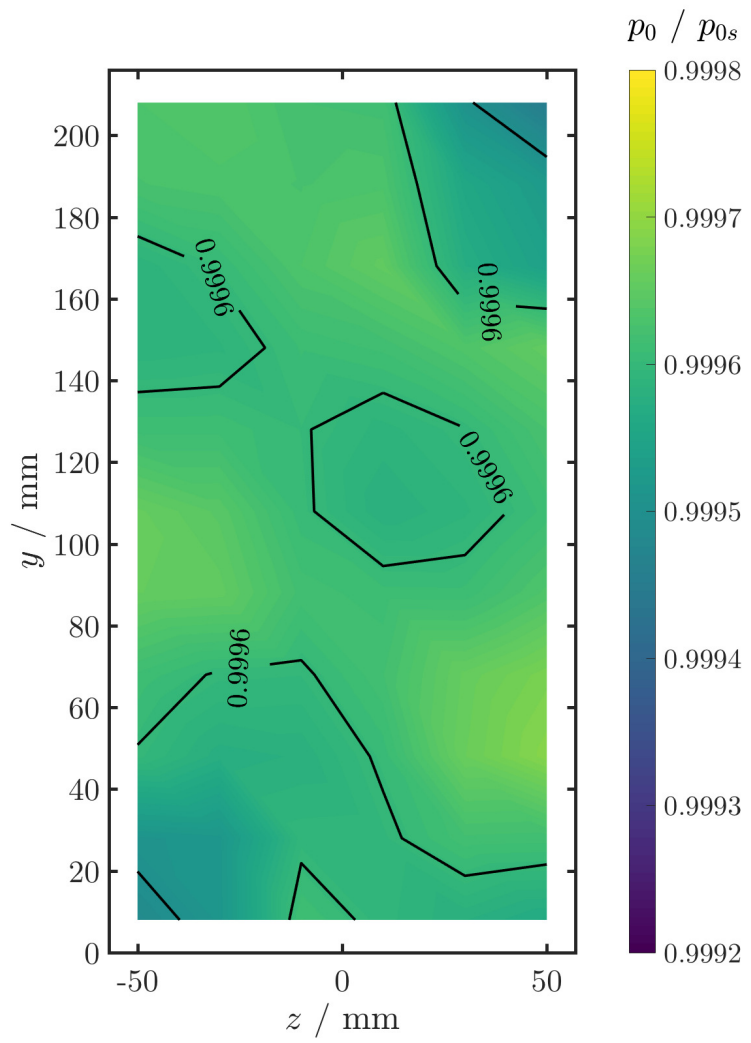

Figure 7. Stagnation pressure measured by Pitot rake at exit of the settling chamber, upsteam of the nozzle. The Pitot probes are located in a square grid, at $20 \mathrm{~mm}$ intervals, between $z=-50$ and $50 \mathrm{~mm}$ and from $y=8$ to $208 \mathrm{~mm}$. 
particles and from the laser optics. Boundary layer traverses are carried out with resolution $\Delta y \approx 0.1 \mathrm{~mm}$. The ellipsoidal probe volume spans $0.1 \mathrm{~mm}$ in the streamwise direction and $2 \mathrm{~mm}$ in the spanwise direction.

The measured boundary layer data is fitted to theoretical profiles (figure 6). A Sun \& Childs (1973) fit, ${ }^{16}$ adapted to include a van Driest compressibility correction, is used for the outer layer; this combines a log-law of the wall region with a Coles wake function. The viscous sublayer is modelled using a Musker (1979) fit. ${ }^{17}$ These fitted profiles are then used to calculate characteristic boundary layer integral parameters. This avoids errors caused by poor measurement resolution near the wall and therefore provides a more accurate estimate of integral boundary layer parameters. The boundary layer properties are determined in their incompressible forms, as these are less sensitive to variations in Mach number and require fewer assumptions to calculate from raw velocity data. The LDV data obtained in this study typically has around 40 measurement points within the boundary layer and the closest data point to the wall is at around $y^{+}=80$. This corresponds to an uncertainty in integral parameters of around $5 \%$ for an equilibrium turbulent boundary layer. ${ }^{18}$

Modifications to the seeding system were required to introduce particles into the corner region, so that LDV measurements could be performed here. The corner seeding system, located upstream of the contraction in the settling chamber, is sketched in figure 4a. Computations of the tunnel flow were used to determine the rake location. Streamlines were traced upstream from the desired measurement region in the working section corners back to the seeding plane in the settling chamber (figure 4b). Placing the rake at this location ensures that seeding particles introduced to the flow pass through the corner region in the working section.

The RANS solver OVERFLOW $2.21^{19}$ is used to model the flow. It uses a Chimera overset mesh technique $^{20}$ in which the Navier-Stokes equations are solved using a third order accurate upwind finite difference scheme using the HLLC model ${ }^{21}$ combined with the Koren limiter. ${ }^{22}$ Turbulence is taken into account using Wilcox $2006 k-\omega$ model. $^{23,24}$ The time integration uses an unfactored SSOR implicit solution algorithm ${ }^{25}$ and multi-grid is utilised to accelerate convergence to a steady state.
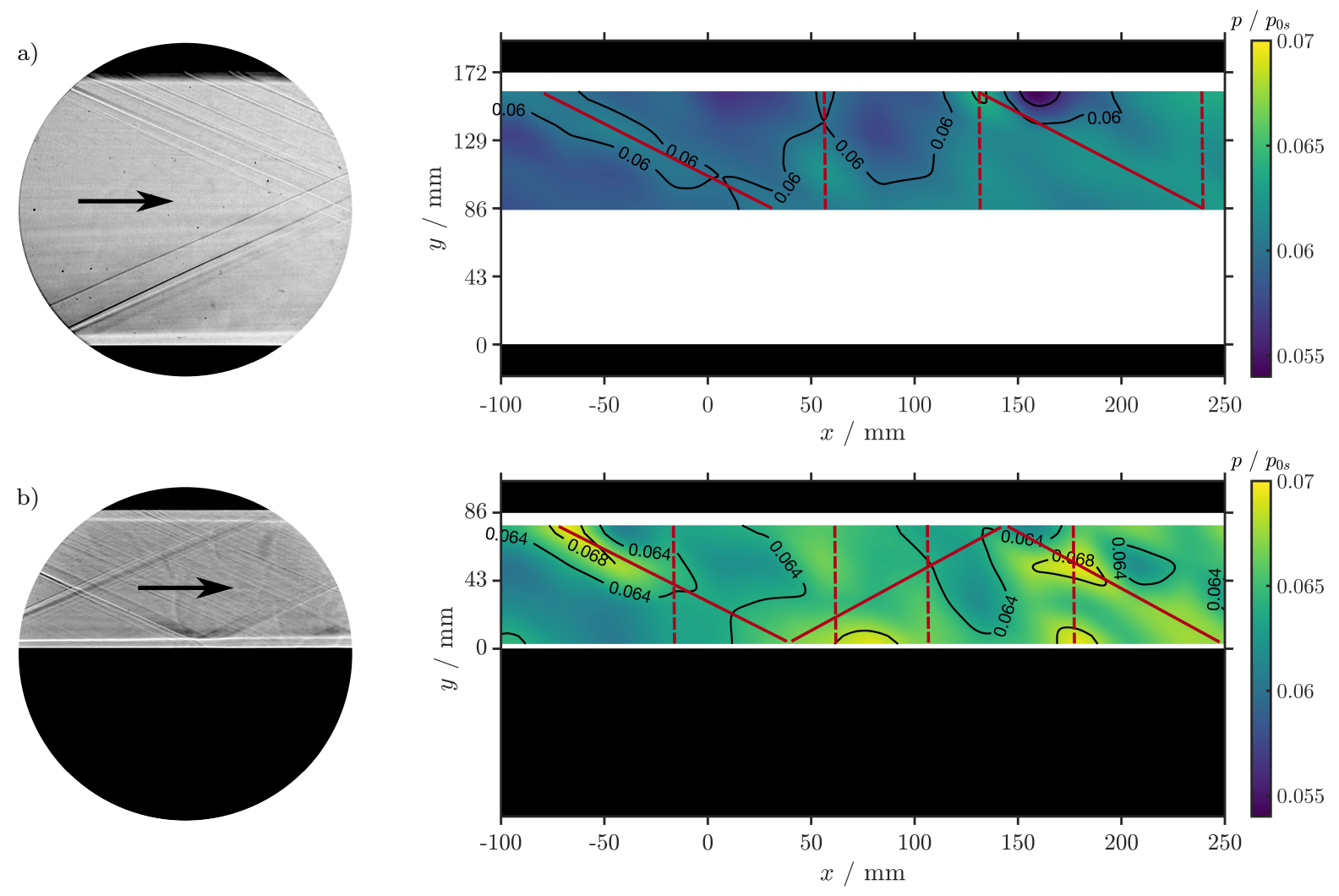

Figure 8. Schlieren images for the tunnel with a) the full setup and b) the half-liner configuration. For each case the measured sidewall static pressure distribution is also presented. The red lines correspond to high-pressure areas; these take the form of oblique (solid) and vertical (dashed) regions. 
The entire tunnel flow is computed from the settling chamber to well downstream of the working section. Only one quarter of the tunnel with the full setup is modelled since it is, in theory, top-bottom and left-right symmetric; similarly, only one half of the tunnel with the half-liner configuration is simulated. An overset mesh methodology was chosen in order to create a smooth mesh in the round-to-square transition region, and around the sharp corner leading to the nozzle blocks. The meshes were generated using Pointwise ${ }^{26}$ mesh generation software. The final mesh contains $181.7 \mathrm{M}$ points across seven blocks. To conduct a mesh resolution study, every other point was removed to create a medium mesh (22.9M points), and every other point was removed from the medium mesh to produce a coarse mesh $(2.9 \mathrm{M}$ points $)$. This method conserves the point distribution across all meshes. A viscous wall spacing of $3 \times 10^{-7} \mathrm{~m}$ was used on the fine grid with a growth rate of $5 \%$, and produced $y^{+}<1$ on all grid levels. The full fine grid is shown in figure 5 .

A nozzle inflow which fixes $p_{0}$ and $T_{0}$, as well as defining the flow angle to be perpendicular to the boundary was used for the inflow; meanwhile, an extrapolated exit condition at the supersonic exit was used. The first few cells along the wall at the inflow were modelled with slip walls to avoid a overdefined boundary condition at the wall. An initial solution was generated using one-dimensional nozzle theory.

A nozzle inflow which fixes $p_{0}$ and $T_{0}$, as well as defining the flow angle to be perpendicular to the boundary was used for the inflow; meanwhile, an extrapolated exit condition at the supersonic exit was used. The first few cells along the wall at the inflow were modelled with slip walls to avoid a overdefined inflow at the wall.

\section{Results and Discussion}

\section{A. Assessment of flow quality}

The stagnation pressure at the exit of the settling chamber, upstream of the nozzle, is surveyed using a Pitot rake. The measured pressure distribution is presented in figure 7 . This shows a highly uniform flow over the tunnel cross-section, with a maximum variation in stagnation pressure across the entire inflow of $0.1 \%$.

Schlieren images of the flow for both nozzle configurations are in figure 8. The boundary layer on the

a)

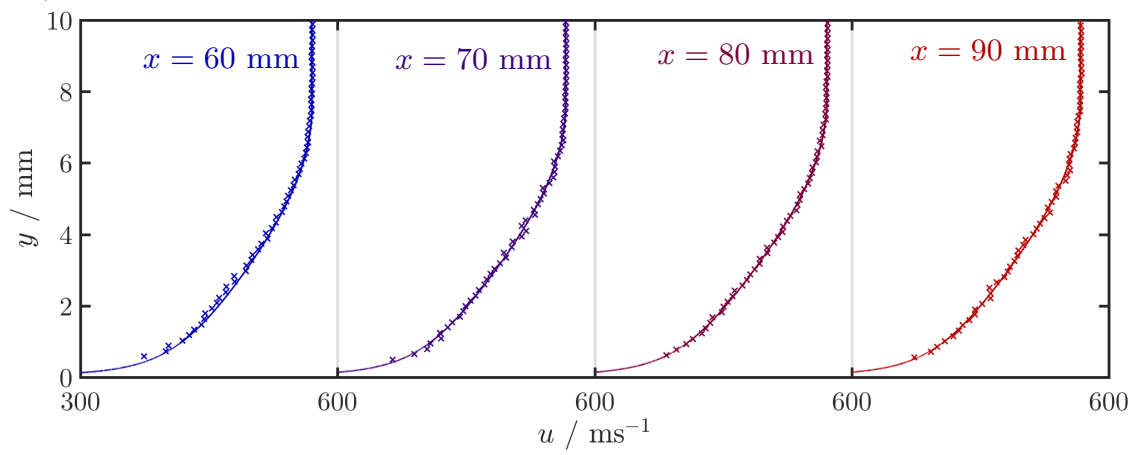

b)

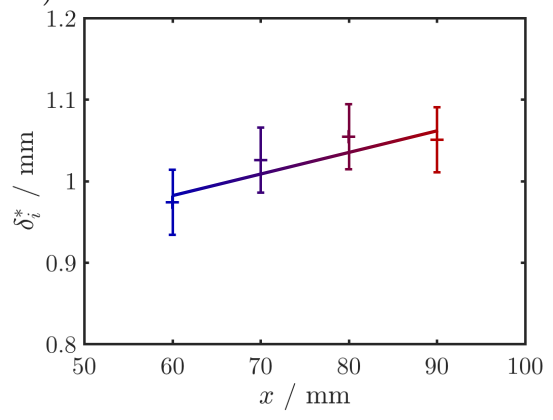

c)

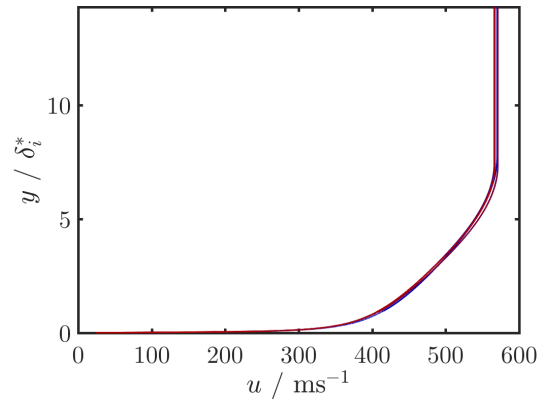

Figure 9. a) Floor boundary layer profiles measured using LDV. These are performed at different streamwise locations on the centre span with the full setup. b) Variation in displacement thickness with streamwise direction. c) Collapse of profile shape when $y$ non-dimensionalised by displacement thickness. 


\begin{tabular}{ccccc}
\hline$x / \mathrm{mm}$ & $\delta / \mathrm{mm}$ & $\delta_{i}^{*} / \mathrm{mm}$ & $\theta_{i} / \mathrm{mm}$ & $H_{i}$ \\
\hline 60 & 7.49 & 0.97 & 0.73 & 1.341 \\
70 & 7.59 & 1.03 & 0.74 & 1.351 \\
80 & 7.70 & 1.05 & 0.76 & 1.356 \\
90 & 7.71 & 1.05 & 0.78 & 1.354 \\
\hline
\end{tabular}

Table 1. Incompressible boundary layer parameters, measured along the centreline with the full setup. These correspond to experimental profiles presented in figure 9a.

a)

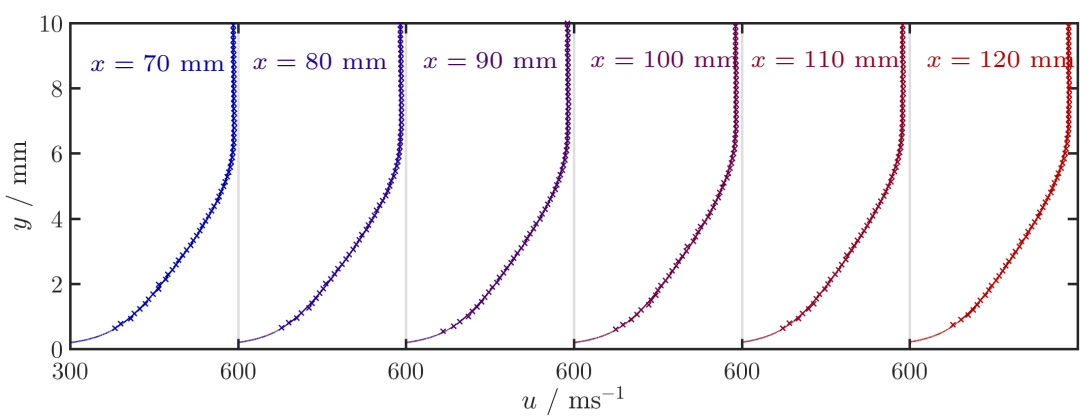

b)



c)

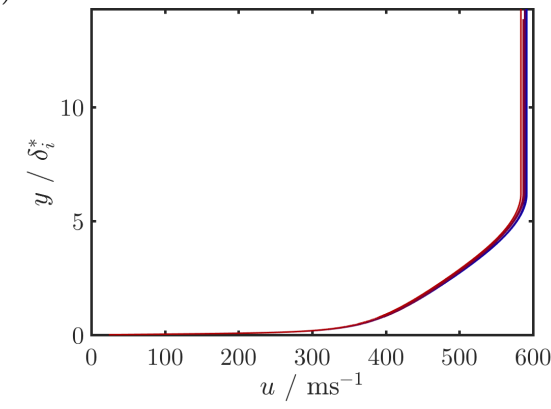

Figure 10. a) Floor boundary layer profiles measured using LDV. Traverses performed at different streamwise locations on the centre span with the half-liner configuration. b) Variation in displacement thickness with streamwise direction. c) Collapse of profile shape when $y$ non-dimensionalised by displacement thickness.

\begin{tabular}{ccccc}
\hline$x / \mathrm{mm}$ & $\delta / \mathrm{mm}$ & $\delta_{i}^{*} / \mathrm{mm}$ & $\theta_{i} / \mathrm{mm}$ & $H_{i}$ \\
\hline 70 & 6.42 & 1.04 & 0.724 & 1.438 \\
80 & 6.53 & 1.07 & 0.740 & 1.439 \\
90 & 6.63 & 1.06 & 0.741 & 1.432 \\
100 & 6.75 & 1.07 & 0.750 & 1.428 \\
110 & 6.87 & 1.11 & 0.772 & 1.433 \\
120 & 6.95 & 1.13 & 0.785 & 1.435 \\
\hline
\end{tabular}

Table 2. Incompressible boundary layer parameters, measured along the centre span with the half-liner, for experimental profiles presented in figure 10a. 


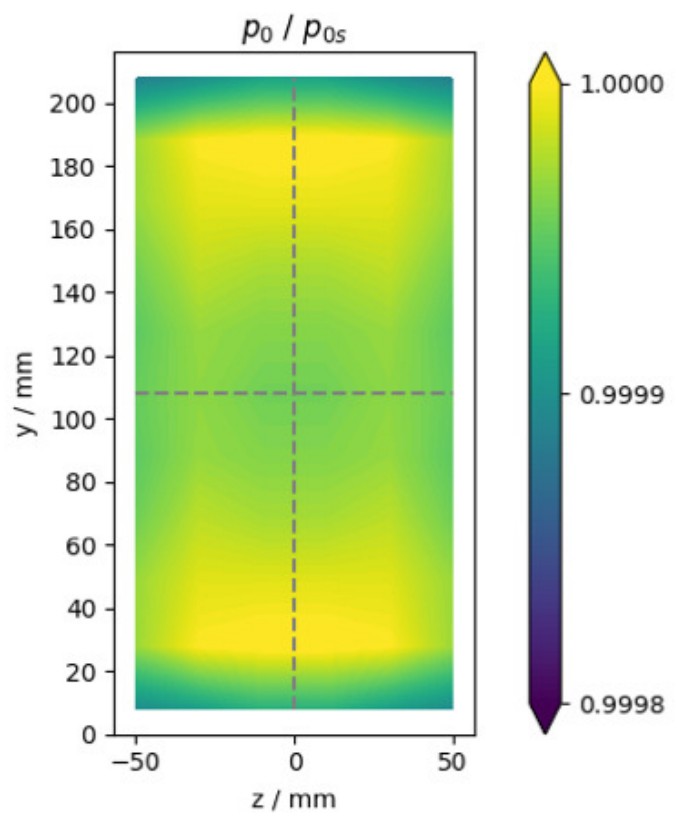

Figure 11. Computed stagnation pressures at the exit of the tunnel contraction interpolated onto the measurement locations.

tunnel floor, as well as weak Mach waves, are visible. The strength of these weak waves are quantified using the static pressure measured using taps along the tunnel sidewall (also shown in figure 8). The data indicates that the static pressure exhibits a variation throughout the working section of $4 \%$ for the full setup and $6 \%$ for the half-liner configuration. These deviations correspond to departures in free-stream Mach number of 0.02 and 0.03 respectively from its mean value of 2.48 . The oblique features in the plots correspond to weak waves generated from the tunnel floor and ceiling, whereas the vertical columns are likely due to spanwise-travelling waves produced by the sidewall.

Figure 9a displays boundary layer profiles measured at different streamwise locations along the tunnel centreline using LDV, for the full setup. The boundary layer parameters corresponding to these profiles are tabulated in table 1. The profile shape is typical for a fully developed turbulent equilibrium boundary layer, with an incompressible shape factor $H_{i} \approx 1.3$. The growth of the boundary layer with streamwise position is also evident. When normalised for boundary layer thickness, as performed in figure $9 \mathrm{c}$, there is a collapse of profiles. Therefore, only very weak waves exist and the profile shape does not vary significantly in this region.

For the half-liner configuration, figure 10a displays boundary layer profiles measured at different streamwise locations along the tunnel centreline; the corresponding boundary layer parameters are presented in table 2. The shape factor of approximately 1.43 corresponds to an equilibrium turbulent boundary layer, though less full than for the full setup (figure 9a). Figure 10b presents the approximately linear growth of the incompressible displacement thickness $\delta_{i}^{*}$. Figure 10c expresses the floor-normal coordinate in terms of boundary layer displacement thickness; the resulting collapse of profiles indicates that the boundary layer is simply growing naturally in the region of interest.

\section{B. Assessment of computations}

To understand how well the computational model accurately represents the physical tunnel flow, it is useful to compare the measured flow characterisation data with equivalent numerical results.

Figure 11 shows the stagnation pressure computed at the end of the tunnel contraction, corresponding to the experimental measurements in figure 7 . In order to reduce any resolution bias, the computed pressures have been interpolated onto the Pitot probe locations. Whilst it appears that the computations predict a lower stagnation pressure loss than was measured in the experiment, the differences are within experimental error. 

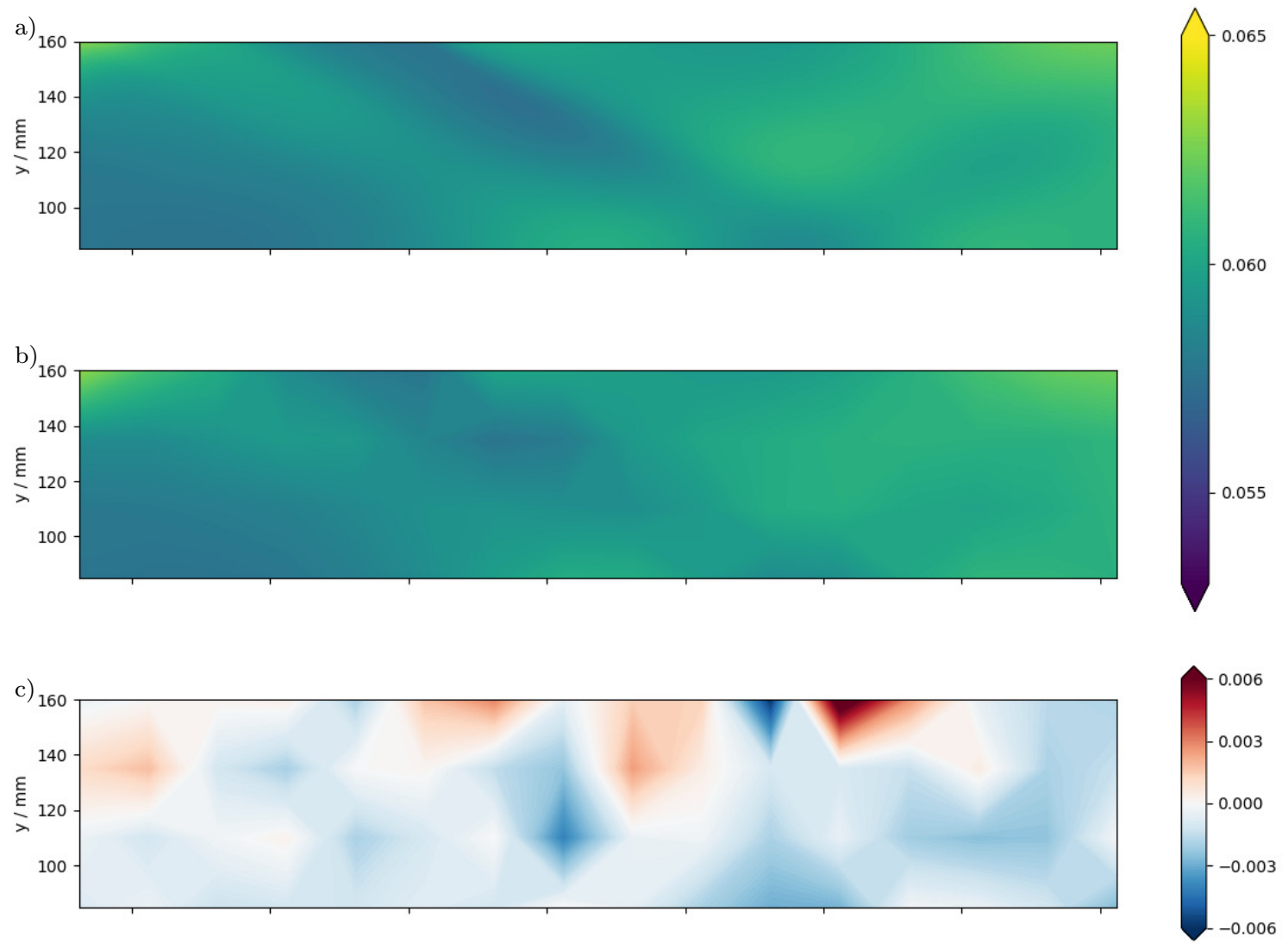

d)

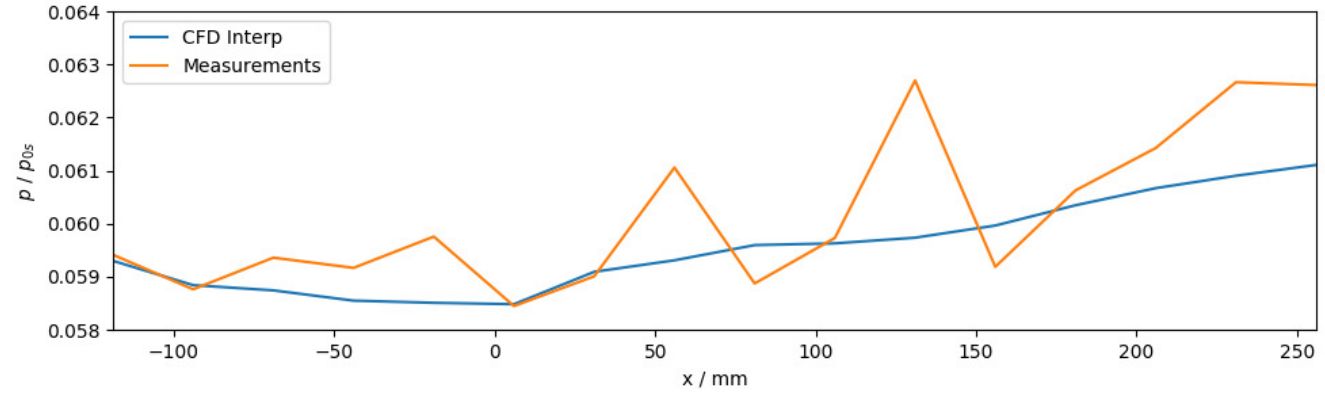

Figure 12. Measured and computed wall static pressures $\left(p / p_{0 s}\right)$ for the full setup. a) full CFD solution b) CFD solution interpolated onto the measurement locations c) difference between the computations and measurements d) measurements and computations averaged over the vertical $y$ direction. 

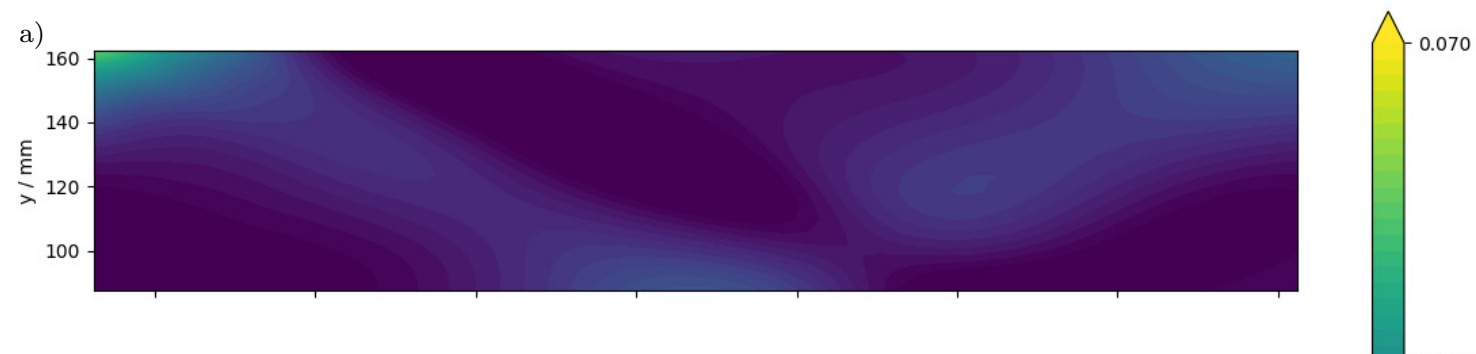

b)
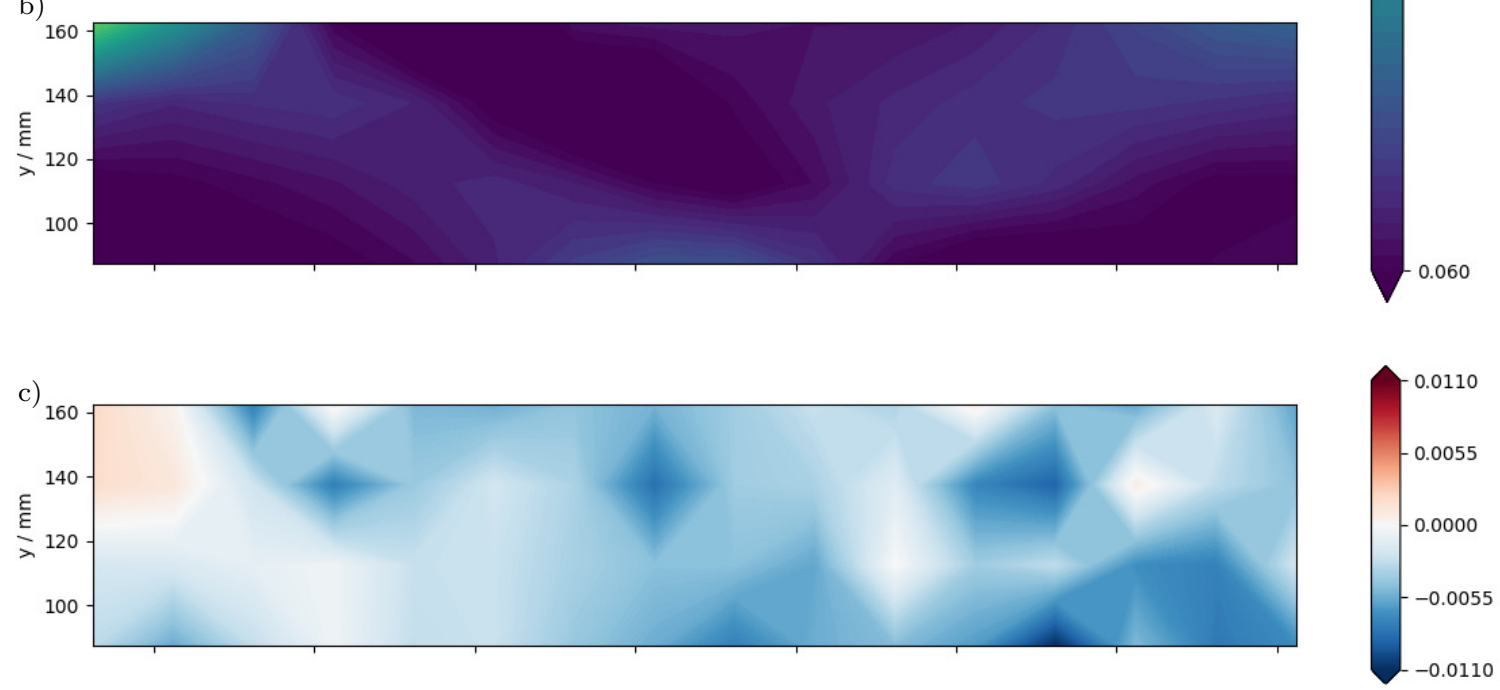

d)

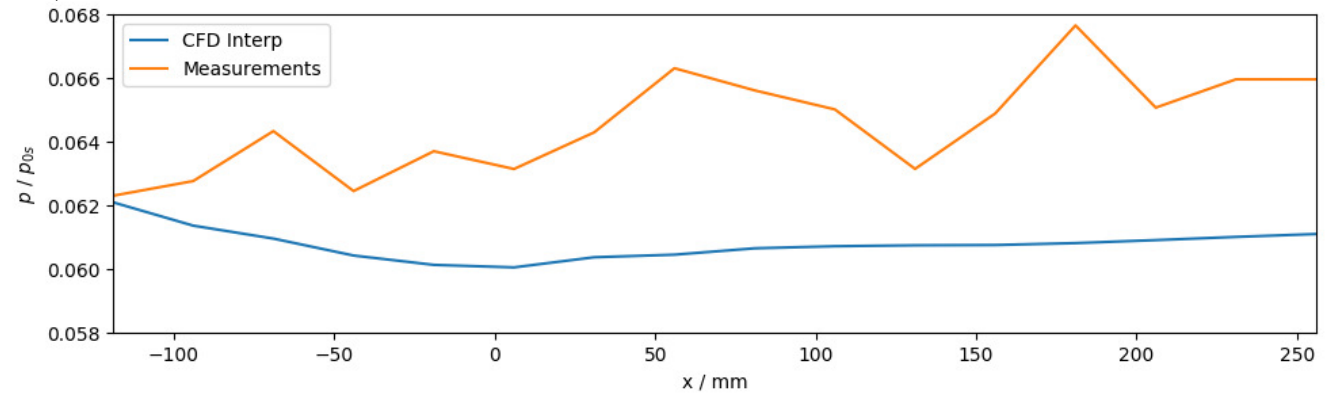

Figure 13. Measured and computed wall static pressures $\left(p / p_{0 s}\right)$ for the half-liner configuration. a) full CFD solution b) CFD solution interpolated onto the measurement locations c) difference between the computations and measurements d) measurements and computations averaged over the vertical $y$ direction. 
a)

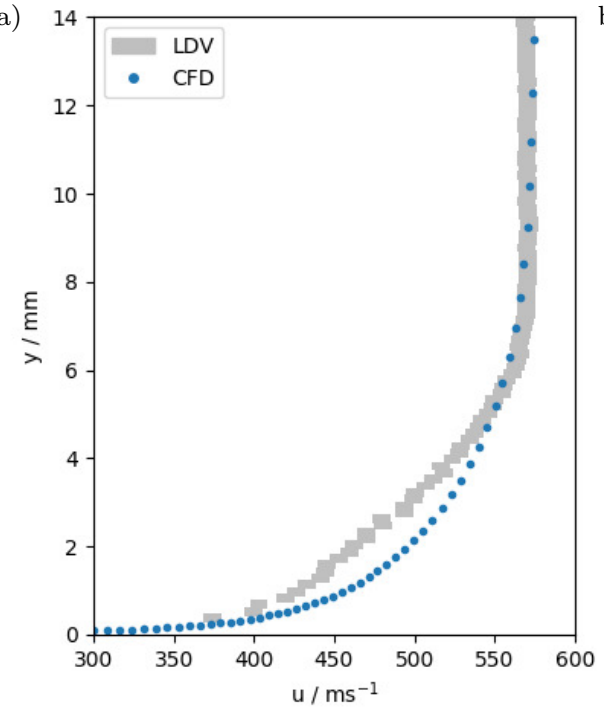

b)

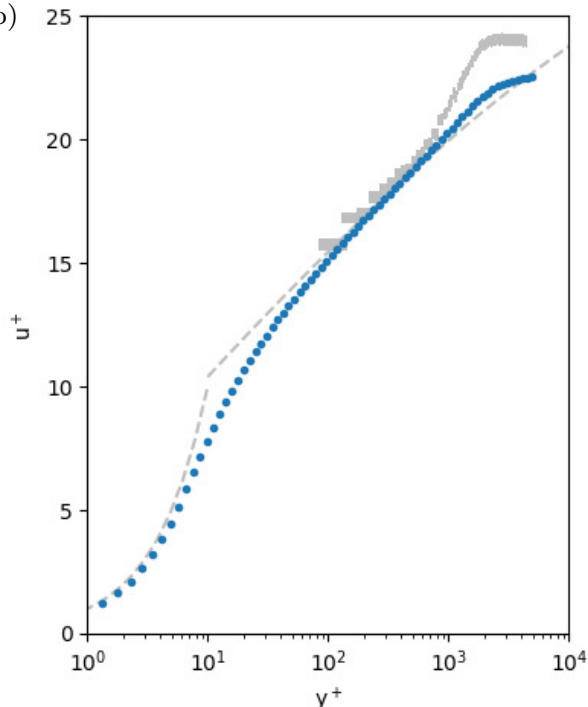

Figure 14. Comparison between computational and experimental floor boundary layer profiles. These are presented for a single measurement station $(x=80 \mathrm{~mm}, z=0 \mathrm{~mm})$ with the full setup. The profiles are presented in both a) dimensional and b) wall units.

A comparison of the measured and computed sidewall static pressures is performed for both configurations. This is shown in figure 12 for the full setup. The uncanceled nozzle wave is visible in both the computations and the measurements. However, the computations do not exhibit the measured vertical waves shown in figure 8. The measurements show a larger pressure rise than the computations over the streamwise extent of this region. It is possible that the vertical waves that are present in the experiment but absent in the computations contribute to this pressure rise.

Meanwhile, figure 13 presents the comparison of sidewall pressure distributions for the half-liner configurations. The computations under-predict the pressures within this region. The reasons for this discrepancy are currently being investigated.

A comparison between computational and experimental floor boundary layer profiles is also performed. A representative example at a single measurement location for the full setup is presented in figure 14. There is good agreement between the freestream velocities between the simulations and the measurements, but the profiles differ in the lower regions of the boundary layer. Investigations are currently being performed in order to determine the source of the discrepancy.

\section{Sidewall secondary flows}

The flowfield over the tunnel cross-section is surveyed using LDV for both setups. A series of traverses are carried out at $x=120 \mathrm{~mm}$. The locations are marked in figure 15a; the spatial resolution of measurements along the lines is $0.075 \mathrm{~mm}$. The streamwise velocities are given in figure $15 \mathrm{~b}$. The tunnel core flow is evident for both configurations, as are the floor, ceiling and sidewall boundary layers. While the floor and ceiling boundary layers have approximately constant thickness across the tunnel span, the sidewall boundary layer thickness varies considerably. In the full setup, the sidewall boundary layers increase in thickness from the corners towards the centreheight; with the half-liner, however, they get thicker from the top to the bottom of the tunnel.

Figure $15 \mathrm{c}$ presents the vertical velocities over the tunnel cross-section. Within the core flow, the vertical velocities do not exceed $1 \%$ of $u$, which is within experimental error. However, there is a significant vertical velocity component ( $5 \%$ of $u$ ) within the sidewall boundary layers, indicative of a secondary flow. The transverse velocities are directed towards the tunnel centre-height in the full setup and in the downwards direction with the half-liner. These will be referred to as sidewall secondary flows. They transport the low momentum boundary layer fluid in the vertical direction, and so affect the sidewall boundary layer thickness. For both configurations, these flows explain the variations in sidewall boundary layer observed in figure 15b. 
a)
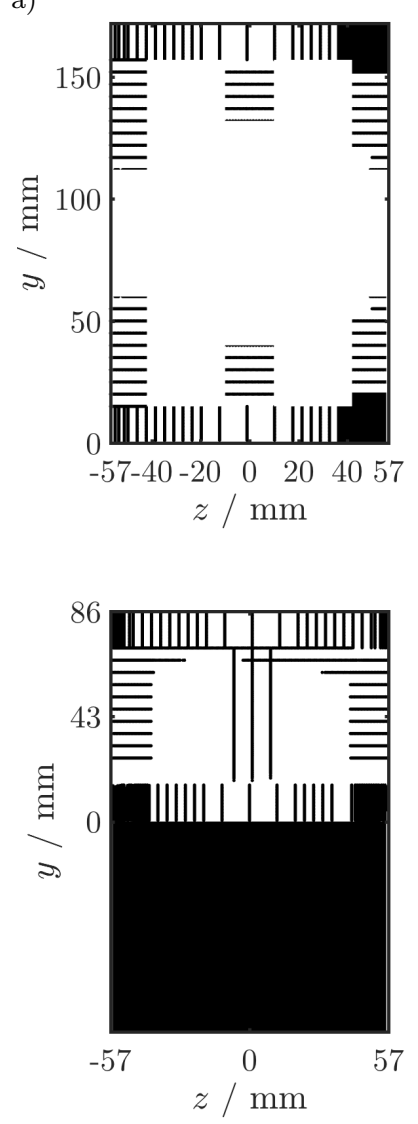

b)
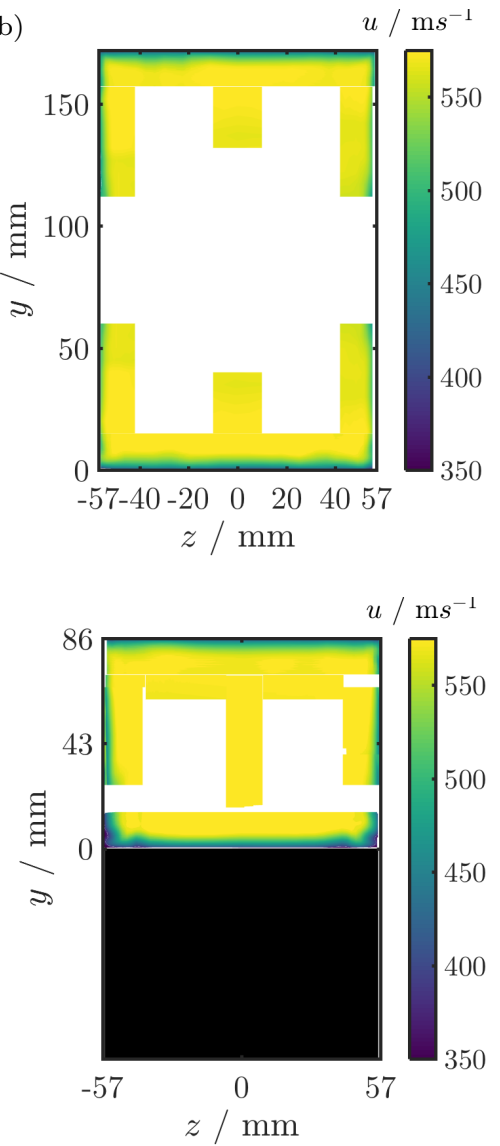

c)



$v / \mathrm{ms}^{-1}$

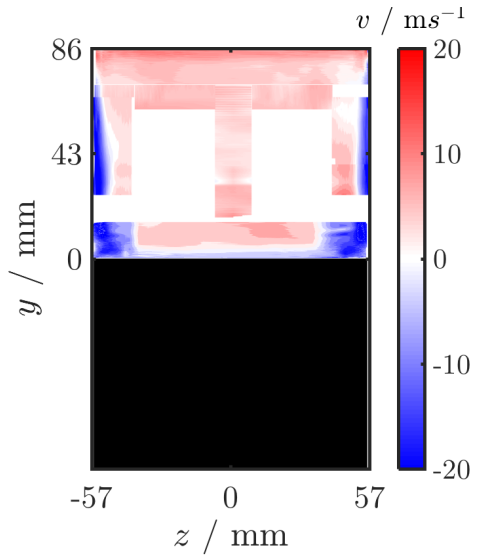

Figure 15. Experimental LDV measurements of the flowfield across the tunnel cross-section at $x=120 \mathrm{~mm}$, for both setups. The plots show a) the traverse locations, b) the streamwise velocity distribution and c) the vertical velocity distribution.

Figure 16 presents the equivalent data from the simulations of the physical tunnel. The streamwise and vertical velocities show the same qualitative features as the experimental measurements from figure 15 . The floor and ceiling boundary layers have constant thickness across the span, while the sidewall boundary layers thicken towards the centre-height of the full setup and bottom corners of the half-liner. Outside the sidewall boundary layer transverse velocities are less than $1 \%$ of $u$; however, within these regions there are significant vertical velocity components ( $5 \%$ of $u$ ). As with the experimental data, these sidewall secondary flows are towards the tunnel centre-height in the full setup and downwards with the full setup. The computational results also provide the spanwise velocity components. The spanwise velocity for both setups does not exceed $0.5 \%$ of $u$ in either setup, indicating that the vertical sidewall boundary layer velocities form the dominant transverse flow within the channel.

The hypothesized mechanism for the sidewall secondary flows is related to the pressure distribution in the nozzle and is depicted schematically in figure 17. We will first consider the full setup. The dashed line in figure 17a.i lies approximately half-way between the nozzle throat and exit. Here, the flow at the centre-height has expanded to the test section pressure while the pressure is higher at the top and bottom of the tunnel. Similar arguments hold elsewhere in the nozzle, since the pressure drop on the curved surface lags behind that at the centre-height. This sets up a vertical pressure gradient (figure 17a.ii). The sidewall boundary layers, with low momentum flow, are most susceptible to this pressure gradient. A secondary vertical flow is therefore introduced in these sidewall boundary layers from the channel corners to the tunnel centre-height (figure 17a.iii). The corresponding mechanism for the half-liner configuration, presented in figure $17 \mathrm{~b}$ is equivalent.

Figure 18a presents the pressure distribution through the nozzle for both configurations, analogous to those in figures figure 17a.i and 17b.i. The resulting cross-sectional pressure distributions, shown in figure 
a) i.

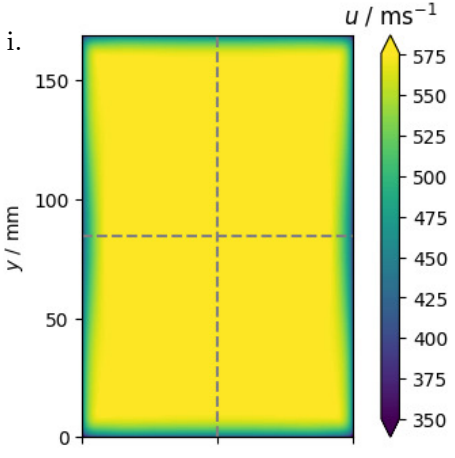

b)

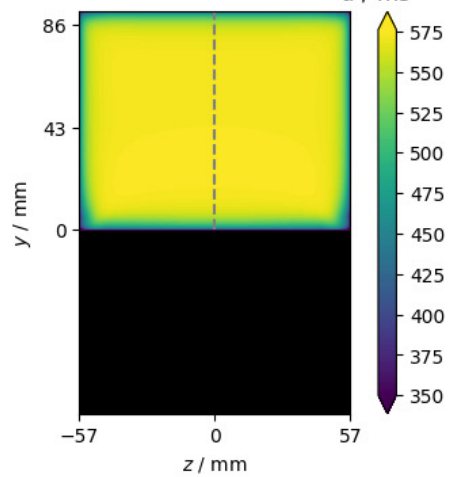

ii.
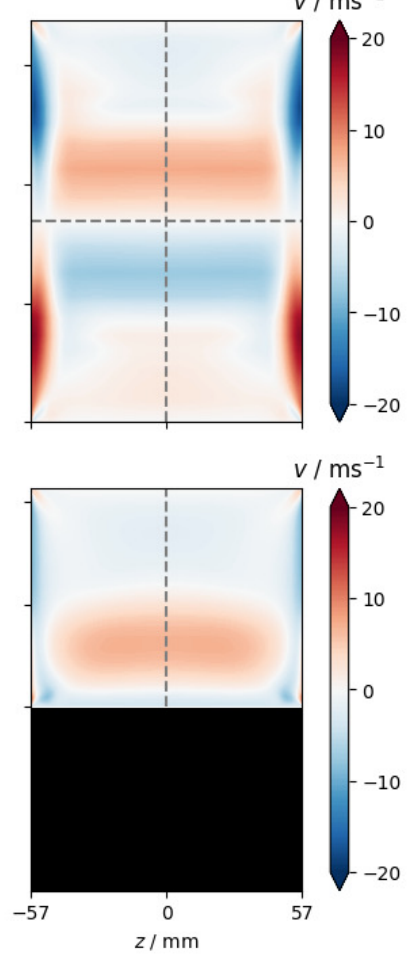

iii.
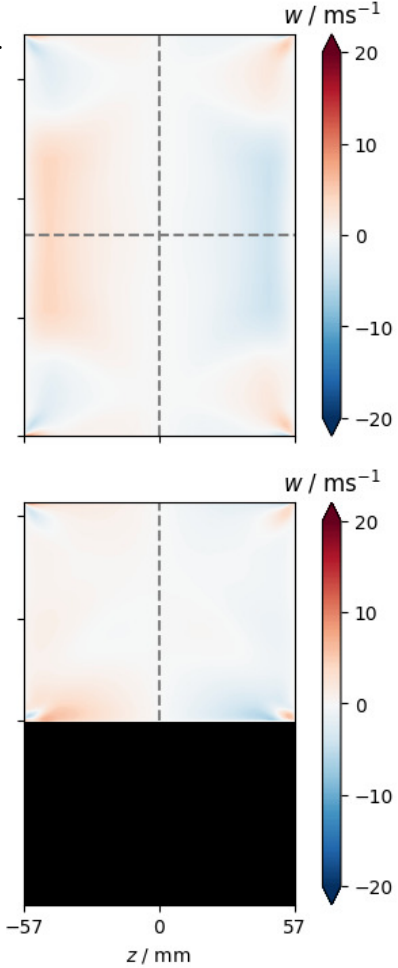

Figure 16. Computational measurements of the flowfield across the tunnel cross-section at $x=120 \mathrm{~mm}$, for a) the full setup and b) the half-liner configuration. The plots show i. the streamwise velocity distribution, ii. the vertical velocity distribution and iii. the spanwise velocity distribution.

a) i.



b) ii.

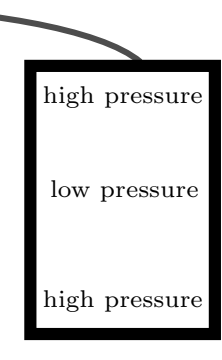

iii.

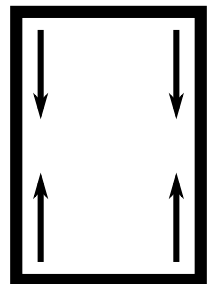

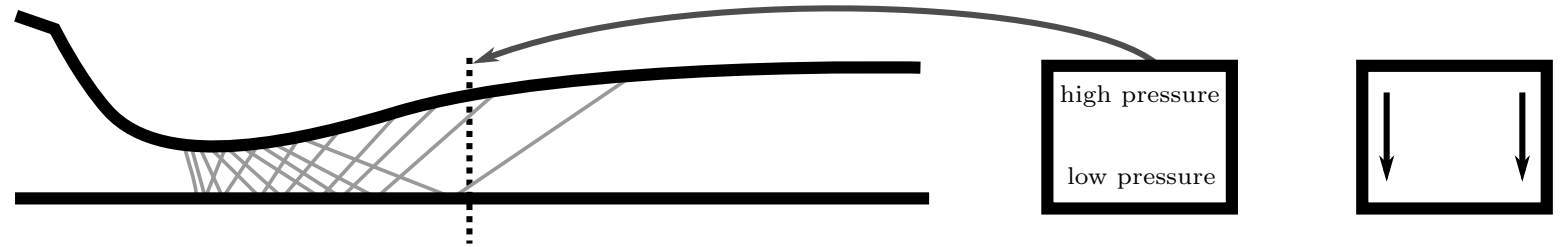

Figure 17. Schematic of the hypothesized secondary flows for a) the full setup and b) the half-liner configuration: i. expansion wave pattern through the nozzle; ii. the cross-sectional pressure distribution upstream of the nozzle exit; iii. induced secondary flows in sidewall boundary layers. 

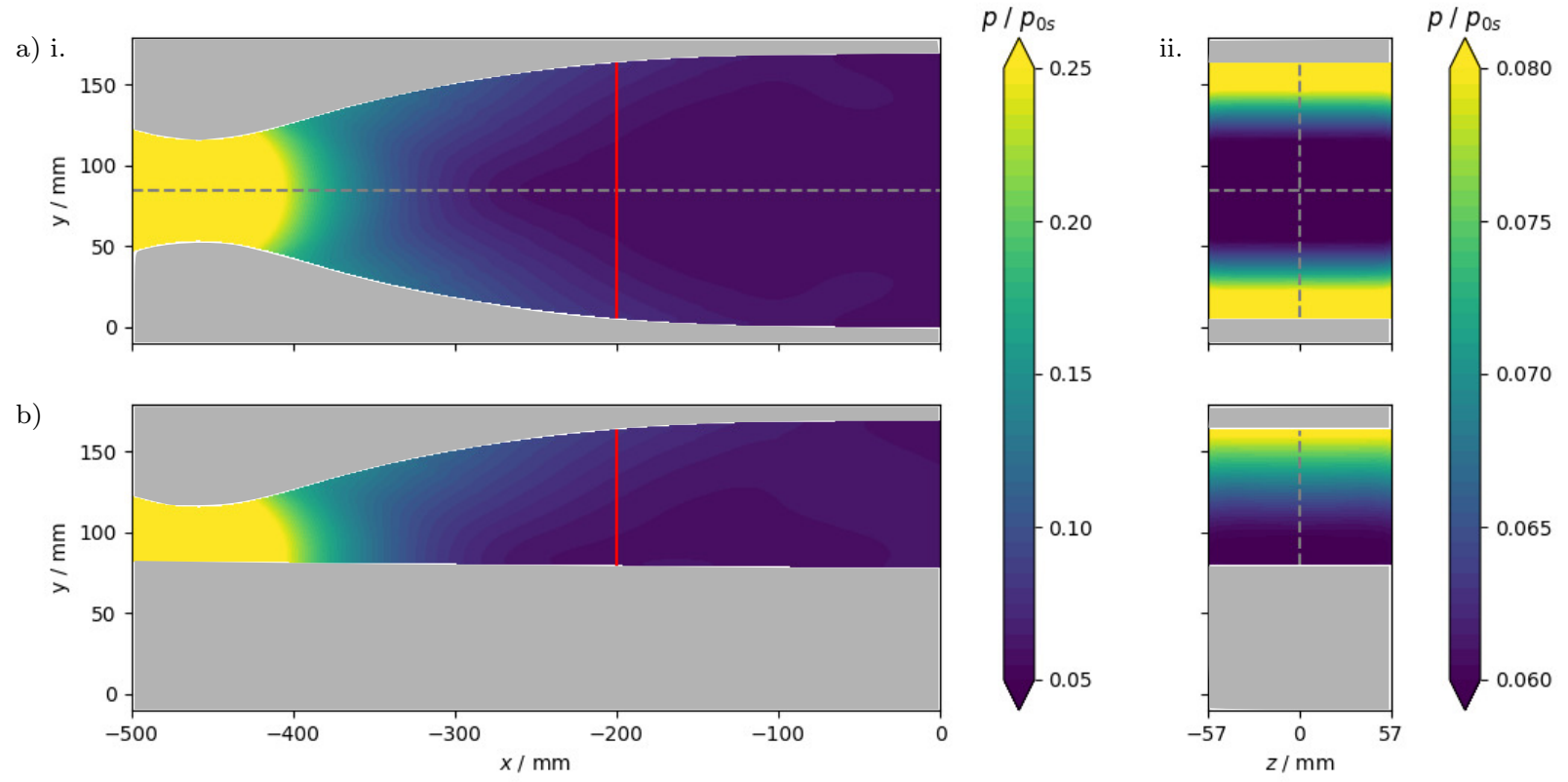

Figure 18. Pressure contours in the nozzle i. on the centre span, and ii. in the $x=-200 \mathrm{~mm}$ plane (corresponding to the red line). These are shown for a) the full setup and b) the half-liner configuration. Note the difference in scale between the plots in $\mathrm{i}$ and ii.

18b, correspond well with those depicted schematically in figures 17a.ii and 17b.ii. This provides some evidence for the generation mechanism of the nozzle-dependent sidewall secondary flows observed in both experiment and computation.

\section{Flow in corner regions}

RANS simulations are known to be poor at computing flows in the corner regions and the method is yet to be validated for this purpose; therefore, analysis of the corner flowfields themselves will be restricted to experimental data. The flowfield in the corner regions is shown for the full setup (figure 19a) and the half-liner configuration (figure 19b). In each figure, the streamwise velocity for all four tunnel corners at $x=120 \mathrm{~mm}$ is presented.

The transfer of high-momentum flow from the core into the corner regions is evident in all cases. This is consistent with the presence of streamwise-aligned vortices in the tunnel corners. The approximate location and sense of these vortices (superimposed on the figure) are inferred from the boundary layers in this region experiencing a local increase in thickness by vortex upwash and entrainment of high-momentum flow by the downwash. There appear to be two distinct flow topologies. All four corners of the full setup and the top corners of the half-liner appear to have a single primary vortex residing on the floor/ceiling, while the bottom corners of the half-liner contain two counter-rotating vortices approximately either side of the corner bisector.

Note that previous studies have measured corner flows in figures $1 \mathrm{~b}$ and $1 \mathrm{c}$ using a full setup and half-liner arrangement respectively. The flowfields measured in the current study agree well with the corresponding flow structures from literature (figures $1 \mathrm{~b}$ and $1 \mathrm{c}$ ) for the two tested configurations. This suggests that the conflicting corner flow topologies measured in previous studies at various facilities can be explained by the installed nozzle geometries.

The data from this study also presents a counter-example to the suggestion by Morajkar et al. that the flow topologies are determined by tunnel aspect ratio. ${ }^{12}$ Although the full setup and half-liner present different aspect ratios, the top corners of the half-liner configuration correspond closely to the corners of the full setup and are quite different to the bottom corners with the half-liner.

Instead, the mechanism affecting corner flowfields appears to be related to the nozzle-dependent sidewall 
secondary flows. Gessner has proposed that, in a supersonic channel with turbulent boundary layers and no other transverse velocities, the Reynolds stresses in the corner boundary layer generate two counterrotating streamwise-aligned vortices, symmetric about the corner bisector. ${ }^{9,27}$ These will be referred to as the sidewall vortex and the floor vortex (figure 20a). Therefore, in the absence of transverse flows, the streamwise vorticity produced in the corner region should result in this ideal, symmetric two-vortex system. However, the nozzle pressure field and induced sidewall secondary flows influence the vortex structure here. While the floor vortex is in a region of weak cross-flow and so is largely unchanged by the sidewall secondary flows, the sidewall vortex is affected by the bulk vertical velocities in this region.

The secondary flows cause advection of any pre-existing sidewall vortex (as well as the continuouslyproduced 'sidewall vorticity') by the local transverse velocity. Let us first consider the cases where the vertical cross flow is directed away from the corners (the full setup and the top corners of the half-liner) - this is shown schematically in figure 20b. Any sidewall vorticity produced near the tunnel sidewall is immediately advected away from the corner by the vertical flow. This prevents the formation of a stable sidewall vortex, and so only the floor vortex remains in the corner region. There is some evidence for this mechanism in the recent experimental investigation by Peltier et al. ${ }^{14}$ performed in a supersonic channel flow with a symmetric nozzle arrangement: the instantaneous flowfields capture a vortex pair, but there is

a)
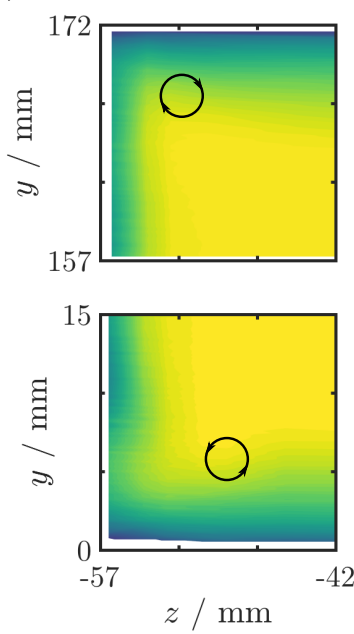



b)
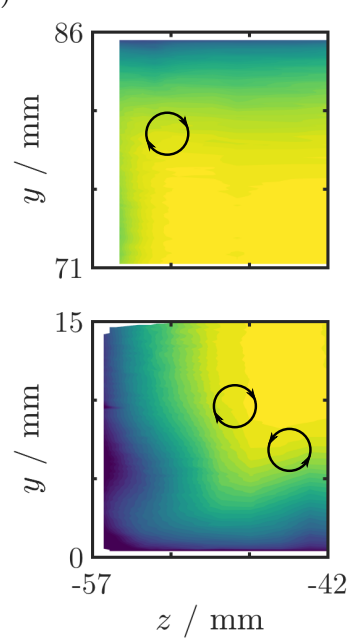

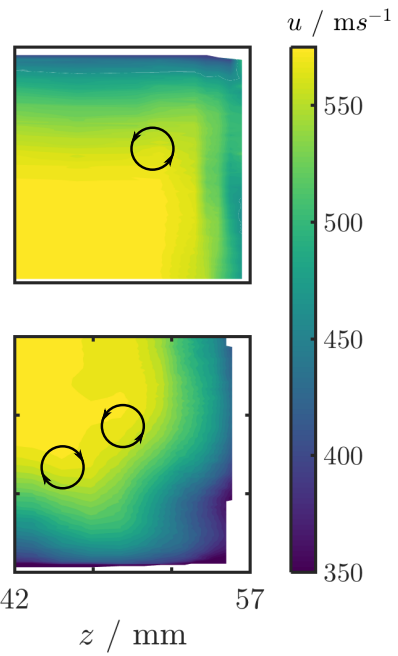

Figure 19. The streamwise velocity distribution measured by LDV at $x=120 \mathrm{~mm}$ in all four tunnel corners for a) the full setup and b) the half-liner configuration. Inferred locations and senses of corner vortices are superimposed.

a)

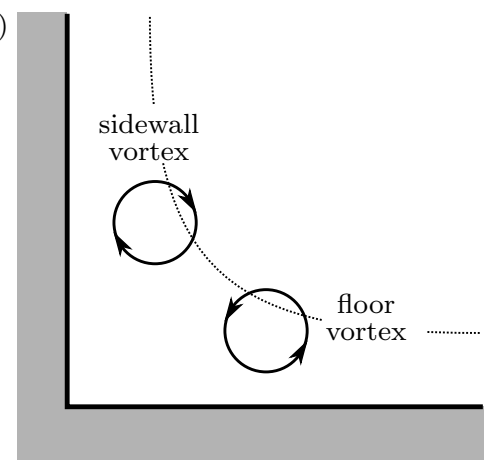

b)

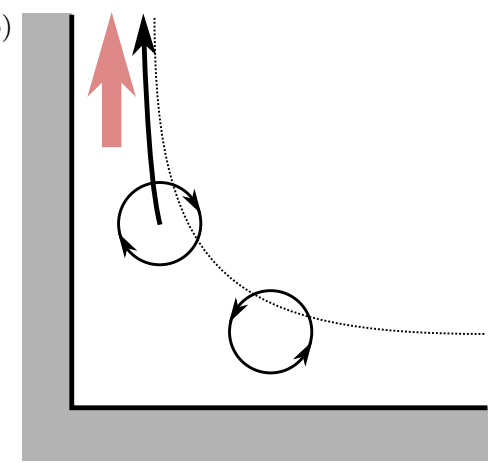

c)

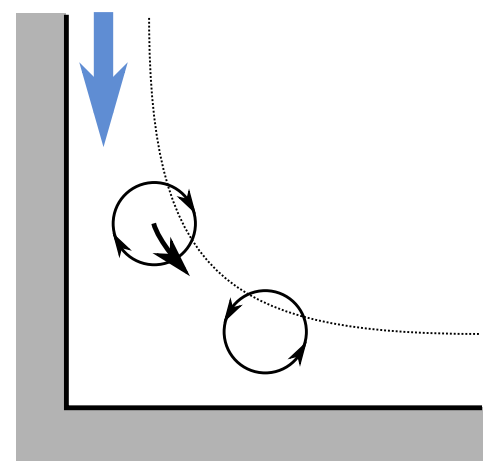

Figure 20. Schematic diagram indicating the effect of sidewall secondary flows on corner vortex structure. The counter-rotating vortex pair with a) no transverse bulk flows, and the influence of vertical sidewall velocities a) away from and b) towards the corner are presented. 
a)

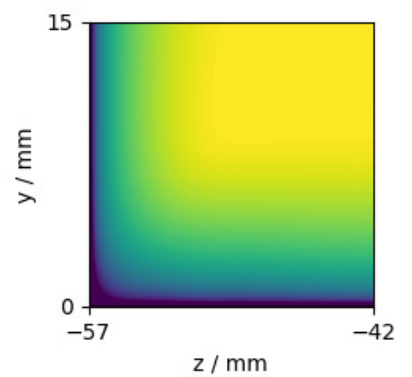

b)

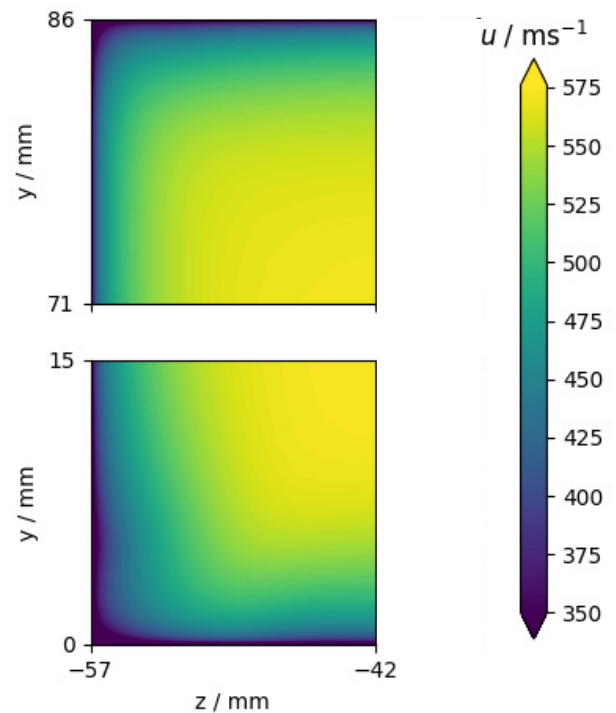

Figure 21. Calculated streamwise velocity in the corner region of a) the full setup and b) the half-liner configuration at $x=120 \mathrm{~mm}$. Note that both computations are left-right symmetric and the full setup is additionally top-bottom symmetric.

only a single floor vortex present in the ensemble-averaged flow (figure 1b).

Figure 20c presents the opposite case, when the sidewall secondary flow is directed into the corner (as for the bottom corners of the half-liner setup). Now, the sidewall vortex is advected towards the corner by the bulk transverse velocity. Therefore, the region retains the counter-rotating vortex pair. The vortex motion induced by the secondary flows does, however, cause the vortex pair to be asymmetric about the corner bisector.

Figures 21a and 21b show the computed corner flow in the full and half-liner setups at $x=120 \mathrm{~mm}$, corresponding to the measurements in figure 19. The computations show some similar features to the experimental results. In particular, the top corners of the half-liner correspond well with the full setup corners while the bottom half-liner corners appear to show a quite different topology. This is consistent with the presented hypothesis of the nozzle geometry influencing the corner flow structure.

However, a direct comparison between figures 19 and 21 shows that the computations do not accurately predict the streamwise velocities in these corner regions. This illustrates the deficiencies of RANS methods in modelling corner flows, and highlights the importance of validation studies to enhance the capabilities of numerical codes.

\section{Conclusions}

This paper presents a joint experimental-computational investigation designed to address a poor understanding of the factors influencing supersonic streamwise corner flows. The study is focused on the flow in an empty shock-free tunnel, without an adverse pressure gradient. The effect of nozzle configurations on corners flows is assessed by operating the tunnel in both a full setup and half-liner configuration.

Careful characterisation of the flow enables quantitative assessment of the flow quality and calibration of computations such that they accurately represent the physical tunnel. The tunnel's cross-sectional pressure distribution upstream of the nozzle is measured to be uniform to within $0.1 \%$. Static pressure measurements enable the strength of extremely weak waves in the flow to be quantified; they cause deviations in the freestream Mach number of less than 0.03 from a mean value of 2.48. For both tunnel setups, the centreline floor boundary layer retains a turbulent equilbrium profile shape while growing naturally in the streamwise direction.

The measurement of flow velocities across the tunnel cross-section enables the identification of vertical velocity components (up to $5 \%$ of the corresponding streamwise velocity) within the sidewall boundary layers. The vertical velocities are directed away from the tunnel corners for the full setup and in the downwards 
direction with the half-liner. These secondary flows are induced by the low-momentum sidewall boundary layers being most susceptible to the vertical pressure gradients set up in the nozzle. These transverse velocity components serve two functions: firstly, they thicken the sidewall boundary layer in the direction of the vertical velocity component by transfer of low-momentum boundary layer fluid; secondly, they affect the flow topology of the corner regions themselves.

All four corners of the full setup and the top corners of the half-liner configuration exhibit a single primary vortex close to the tunnel floor or ceiling. Meanwhile, the bottom half-liner corners appear to contain a counter-rotating vortex pair, albeit not symmetric about the corner bisector.

In the absence of transverse velocities, a symmetric counter-rotating vortex pair would be generated in each corner boundary layer by the Reynolds stresses. The observed corner flowfields are explained by considering modifications to this ideal vortex structure due to the sidewall secondary flows. The vertical advection of vorticity close to the sidewall by the bulk cross-flow results in a single primary floor vortex in the first case and a steady counter-rotating vortex pair in the second. Since the secondary flows are directed away from the tunnel corners in the full setup and downwards with the half-liner, the described process explains the differences between the two corner flow topologies measured in this study. Since this mechanism is also consistent with the various corner vortex structures measured in other studies, it appears as though the nozzle geometry is the dominant cause of the observed differences between them.

The separation properties of the corner boundary layer under an adverse pressure gradient are dependent on the momentum in the corner region. This is influenced by the thickness of the sidewall boundary layer and by the properties of the vortices which entrain high-momentum core flow into the corner. Both these factors are intimately associated with the sidewall secondary flows; however their relative importance is yet to be established.

In conclusion, this study has identified the presence of sidewall secondary flows, which take distinct forms for the two nozzle geometries tested. The mechanism for the generation of these transverse flows is based on the pressure distribution in the nozzle. These vertical velocities within the sidewall boundary layers are responsible for modifying the vortex topology in the corner and thus the momentum in this region. The prevalence of two-dimensional nozzles within supersonic wind tunnel setups means that sidewall secondary flows are almost ubiquitous. Since they apparently have a significant influence on corner flows, experimental studies on these regions should consider the effects of these transverse velocities. Futhermore, when validating numerical codes using experimental data, the corner flowfield itself cannot be accurately computed without a knowledge of the nozzle geometry, and thus the sidewall secondary flows.

\section{Acknowledgments}

The authors would like to thank David Martin, Sam Flint, Anthony Luckett and Ciaran Costello for operating the blowdown wind tunnel. The wind tunnel is part of the UK National Wind Tunnel Facility (NWTF) and their support is gratefully acknowledged. The research leading to these results has received funding from the Air Force Research Laboratory.

\section{References}

${ }^{1}$ J. Benek. Corner flows: motivation \& objectives. Comments made at the 10th Annual Shock Wave/Boundary Layer Interaction (SWBLI) Technical Interchange Meeting, 2017.

${ }^{2}$ N. Titchener and H. Babinsky. Shock wave/boundary-layer interaction control using a combination of vortex generators and bleed. AIAA Journal, 51(5):1221-1233, 2013.

${ }^{3} \mathrm{H}$. Babinsky, J. Oorebeek, and T. Cottingham. Corner effects in reflecting oblique shock-wave/boundary-layer interactions. In 51st AIAA Aerospace Sciences Meeting including the New Horizons Forum and Aerospace Exposition, 2013-0859.

${ }^{4}$ R.M. Chriss, W.R. Hingst, A.J. Strazisar, and T.G. Keith. An LDA investigation of three-dimensional normal shock wave boundary-layer interactions. In Proceedings of Transonic Symposium: Theory, Application and Experiment, volume 1, pages 741-764, 1989 .

${ }^{5}$ P.J. Bruce, H. Babinsky, B. Tartinville, and C. Hirsch. Corner effect and asymmetry in transonic channel flows. AIAA Journal, 49(11):2382-2392, 2011.

${ }^{6}$ J. Benek, C. Suchyta, and H. Babinsky. The effect of tunnel size on incident shock boundary layer interaction experiments. In 51st AIAA Aerospace Sciences Meeting including the New Horizons Forum and Aerospace Exposition, $2013-0862$.

${ }^{7} \mathrm{X}$. Xiang and H. Babinsky. Corner effects in oblique shock wave/boundary layer interactions in rectangular channels. In 55th AIAA Aerospace Sciences Meeting, 2017-0984.

${ }^{8} \mathrm{X}$. Xiang and H. Babinsky. An experimental study of corner flow control applied to an oblique shock-wave/boundary-layer interaction. In 56th AIAA Aerospace Sciences Meeting, 2018-1532. 
${ }^{9}$ F.B. Gessner, S.D. Ferguson, and C.H. Lo. Experiments on supersonic turbulent flow development in a square duct. AIAA Journal, 25(5):690-697, 1987.

${ }^{10}$ D.O. Davis and F.B. Gessner. Further experiments on supersonic turbulent flow development in a square duct. AIAA Journal, 27(8):1023-1030, 1989.

${ }^{11}$ D.O. Davis, F.B. Gessner, and G.D. Kerlick. Experimental and numerical investigation of supersonic turbulent flow through a square duct. AIAA Journal, 24(9), 1986.

${ }^{12}$ R.R. Morajkar, J.F. Driscoll, and M. Gamba. Experimental study of supersonic turbulent corner flow evolution in a low aspect ratio rectangular channel. In 53rd AIAA Aerospace Sciences Meeting, 2015-0542.

${ }^{13}$ B.E. Rice, N.J. Bisek, S.J. Peltier, and J.W. Hofferth. Investigation of secondary motion in high speed flow. In $55 t h$ AIAA Aerospace Sciences Meeting, 2017-0526.

${ }^{14}$ S.J. Peltier, B.E. Rice, N.J. Bisek, C.K. McKenna, and J.W. Hofferth. Structure of secondary motion in a Mach 2 boundary layer. In 2018 AIAA Aerospace Sciences Meeting, 2018-0583.

${ }^{15}$ S.P. Colliss, H. Babinsky, K. Nübler, and T. Lutz. Vortical structures on three-dimensional shock control bumps. AIAA Journal, pages 2338-2350, 2016.

${ }^{16}$ C.C. Sun and M.E. Childs. A modified wall wake velocity profile for turbulent compressible boundary layers. Journal of Aircraft, 10(6):381-383, 1973.

${ }^{17}$ A.J. Musker. Explicit expression for the smooth wall velocity distribution in a turbulent boundary layer. AIAA Journal, 17(6):655-657, 1979.

${ }^{18}$ N. Titchener, S. Colliss, and H. Babinsky. On the calculation of boundary-layer parameters from discrete data. Experiments in Fluids, 56(8):159, 2015.

${ }^{19}$ P.G. Buning, D.C. Jespersen, T.H. Pulliam, W.M. Chan, J.P. Slotnick, S.E. Krist, and K.J. Renze. Overflow user's manual. NASA Langley Research Center, Hampton, VA, 2002.

${ }^{20}$ J. Benek, J. Steger, and F.C. Dougherty. A flexible grid embedding technique with application to the Euler equations. In 6th Computational Fluid Dynamics Conference Danvers, page 1944, 1983

${ }^{21}$ E.F. Toro, M. Spruce, and W. Speares. Restoration of the contact surface in the HLL-Riemann solver. Shock Waves, 4(1):25-34, 1994.

${ }^{22} \mathrm{~B}$. Koren. Upwind schemes, multigrid and defect correction for the steady Navier-Stokes equations. In 11th International Conference on Numerical Methods in Fluid Dynamics, pages 344-348. Springer, 1989.

${ }^{23}$ D.C. Wilcox. Reassessment of the scale-determining equation for advanced turbulence models. AIAA Journal, 26(11):1299-1310, 1988.

${ }^{24}$ D.C. Wilcox. Turbulence Modeling for CFD. DCW Industries, Inc., 5354 Palm Drive La Canada, CA 91011, 3 edition, 2006.

${ }^{25} \mathrm{R}$. Tramel and R. Nichols. A highly efficient numerical method for overset-mesh moving-body problems. In 13 th Computational Fluid Dynamics Conference, page 2040, 1997.

${ }^{26}$ Pointwise Version. 18.0R4. Pointwise Inc., Fort Worth, TX, 2017.

${ }^{27}$ F.B. Gessner. The origin of secondary flow in turbulent flow along a corner. Journal of Fluid Mechanics, 58(1):1-25, 1973. 